\title{
Los signos del mal y la cultura popular en Los vivos $y$ los muertos de Edmundo Paz Soldán
}

\author{
Karim Benmiloud \\ Professeur à l'Université de Montpellier 3
}

Karim Benmiloud envisage d'aborder la représentation littéraire du Mal dans Los vivos y los muertos à partir de l'omniprésence de la mort, paroxysme du Mal. Il tente, dans une féconde exploration du roman, d'en décrypter les signaux qui, selon lui, sont reliés autant à une tradition littéraire et artistique américaine, qu'à une culture contemporaine populaire, américaine également, toute de musiques à la mode, de nouvelles technologies, de films cultes et de sport. Karim Benmiloud s'attache à souligner les références à une société moderne extrêmement codifiée ainsi qu'à un espace imaginaire, mais clairement identifié comme la représentation fictive d'une petite ville universitaire américaine de l'upstate New York, paradigmatique d'un espace où les clichés, nombreux et variés, s'accumulent : les films de série $B, l^{\prime}$ évocation de James Dean, jeune et célèbre, également mort d'un accident de voiture comme les frères jumeaux du roman, la célébration de la fête d'Halloween, le football américain. Toutes ces références sont autant d'évocations d'une Amérique stéréotypée que Paz Soldán convoque dans son roman pour les mettre au service de tragédies aux multiples visages. Plus particulièrement, le cinéma nordaméricain mais également la musique pop et le sport s'immiscent dans le roman, dans l'intrigue même et chez les personnages afin de fonder une nouvelle représentation du Mal, nourrie de mythes modernes qui empruntent davantage à la culture populaire américaine qu'à une tradition savante et universelle. Karim Benmiloud insiste, pour conclure, sur la subtilité d'une écriture qui joue et se joue de ces références multiples et évidentes, donnant ainsi naissance à un récit d'une complexité narrative originale.

Bolivie, Edmundo Paz Soldán, Los vivos y los muertos, roman, la mort, le mal, culture populaire, tradition littéraire, espace imaginaire, clichés, stéréotypes, mythes modernes

«Pobre [lector]. Pero no es pobre, es un idiota por no leer las señales a tiempo » ${ }^{1}$

Como lo sugiere el mismo título, la novela de Edmundo Paz Soldán titulada Los vivos y los muertos, nos propone un trayecto y un recorrido, de la vida a la muerte, y de Los vivos (a) los muertos, ensanchando cada vez más el radio de acción de la muerte en la desgraciada existencia de los habitantes de la ciudad de Madison, Estados Unidos. Y la muerte se impone no sólo para interrumpir, cortar y abreviar tajantemente la vida de algunos de sus jóvenes (y menos jóvenes) habitantes, sino también para impactar en forma definitiva la vida de los sobrevivientes, sumiéndolos en el más patético desconsuelo, en el más profundo dolor, del que nunca lograrán salir del todo, por la misma violencia y crueldad de los asaltos sufridos.

Uno de los aspectos más interesantes que cobra la omnipresencia progresiva de la muerte en la economía de la novela es, a nuestro juicio, la frecuencia y la intensidad con las que se manifiestan los signos del Mal por venir, mediante una serie de acotaciones a primera vista inocentes e intrascendentes, que acaban sin embargo por revelarle al lector un oscuro camino de la fatalidad hacia el imperio del Mal y de la Muerte.

Uno de los aciertos más loables de la novela es, a nuestro parecer, la forma original que cobran estos signos del Mal, recuperando y renovando el novelista a la vez una tradición muy

\footnotetext{
${ }^{1}$ Edmundo Paz Soldán, Los vivos y los muertos, Madrid, Alfaguara, 2009, p. 125 (todas las citas se harán de esta edición).
} 
marcada de la narrativa o del relato estadounidense (novela negra, hard boiled, no-ficción, etc.) y, sobre todo, una serie de elementos de la cultura popular que habían sido desechados hasta hace poco por la novela, aunque formen parte del acervo cultural más reciente de nuestra cultura contemporánea occidental, profundamente influida por la subcultura popular, el cine, el rock y la música pop, la publicidad, los videojuegos, internet, etc.

Por ser la novela enfocada desde los puntos de vista de once narradores distintos, que se expresan en primera persona (mediante el fluir de conciencia), la mayor parte de estos referentes populares son los de los principales protagonistas de la novela, o sea los siete adolescentes Tim, Amanda, Jem, Hannah, Yandirah, Colin el Enterrador, y Rhonda (y en menor grado el niño Junior). Dado, pues, que los personajes de la novela vienen conformados -íntima y sicológicamente- por la cultura promovida, difundida e impuesta en gran escala desde mediados de los 50's por los mass media estadounidenses, o sea el cine, la música, los cómics, la publicidad, el deporte, y desde los 80's y 90's los videojuegos e internet ${ }^{2}-0$ sea toda una industria de la comunicación y del entertainment-, no es de extrañar que sus ocupaciones, sus representaciones y su imaginario dejen poco espacio a la cultura clásica tradicional, la que ha ido conformando desde hace siglos el trasfondo obligatorio de la novela decimonónica. Como lo dice acertadamente Giovanna Rivero en un brillante artículo, « Es la amalgama de manufactura narrativa (referencias musicales, ropa, indicios generacionales, uso de la tecnología) con algunas leyendas urbanas tan propias del folk de las vastas ciudades universitarias norteamericanas [...], lo que hace de esta novela un ejemplar Kitsch $»^{3}$. Es lo que observa la misma Amanda -no en vano la que tiene la relación más elaborada y más estrecha con la escritura- en la segunda parte de la novela:

El mundillo joven de Madison no ha tenido oportunidades para construir su mitología. Ha vivido precariamente el momento, soñando con un James Dean capaz de rescatarlo, perdiéndose en videojuegos para oligofrénicos, reality shows imbéciles y MTV. (p. 130)

Como puede verse, en el mejor de los casos, la única auténtica cultura de la que pueden valerse los adolescentes protagonistas de la novela, es una tímida e incipiente cultura cinematográfica, pero teñida una vez más con el aura tenebrosa de la muerte prematura y mediática (nos referimos al accidente de James Dean). Así veremos cómo, poco a poco, nuevos códigos y nuevos referentes van sustituyendo ineluctablemente las mitologías y los códigos literarios anteriores. A partir de esta observación, iremos cotejando los elementos e indicios sucesivos que van anunciando al lector, mediante un nuevo lenguaje y nuevas representaciones, el Mal y la Muerte con la que la literatura siempre ha estado compitiendo y lidiando desde los orígenes.

\section{Érase una vez... los cuentos de hadas}

En un principio, sin lugar a dudas, la psique humana de los jóvenes protagonistas sigue conformada por los relatos infantiles, es decir los jugosos cuentos de hadas a los que se abrevaron de niños, como las generaciones anteriores (occidentales o no) lo hicieron antes de ellos. Por lo cual, y también por la misma juventud de los principales protagonistas de la novela, el cuento infantil sigue teniendo la misma función de advertencia e iniciación para los niños y jóvenes adolescentes de la novela, que tendrán que enfrentarse con el Mal y la Muerte de forma inesperada y violenta.

No es de extrañar por lo tanto que el primer capítulo empiece y acabe con un semáforo rojo, señal de advertencia en el código de la circulación, y señal simbólica de la prohibición

\footnotetext{
${ }^{2}$ Sobre este tema en tres novelas recientes (entre las cuales Río Fugitivo de Edmundo Paz Soldán), véase el artículo de Jesús Montoya Juárez, « Ni apocalípticos ni integrados: medios audiovisuales en tres narradores del sur de América », Revista Iberoamericana, vol. LXXIII, núm. 221, oct.-dic., 2007, pp. 887-902.

${ }^{3}$ Giovanna Rivero, «El crimen Kitsch », en Érich Fisbach (coord.), Tradition et modernité dans l'œuvre d'Edmundo Paz Soldán, Angers, Presses de l’Université d’Angers, 2010, 169-174, p. 170.
} 
de la transgresión, que el personaje de Tim, primer narrador, cometerá para su desgracia, con la llegada de un fatal accidente de tráfico. No es de extrañar, sobre todo, que esta señal de advertencia se desdoble al final del capítulo con una alusión al famoso cuento de hadas Caperucita roja, que, en los segundos anteriores al accidente, le avisan al lector de la inminencia del peligro y de la sombra de una amenaza fatal:

Amanda: [...]. Cuando me dijo qué ojos más verdes que tienes, y yo le dije para verte mejor. Cuando me dijo qué nariz más recta que tienes, y yo para olerte mejor. Cuando me dijo qué labios más grandes que tienes, y yo para comerte mejor, y me dijo qué esperas, esta Caperucita Roja está lista para que se la coman y dejamos de ver la película. (p. 17)

Como puede verse, el recuerdo del cuento sirve aquí para pasar del juego inocente de los niños a la relación carnal de los jóvenes amantes (la escena se desarrolla en un hotel de la Ruta15, la misma del accidente que tendrá lugar segundos después de la irrupción de dicho recuerdo en la mente de Tim que está manejando su auto). De hecho, la novela introducirá después una figura paradigmática del Mal con el personaje del violador-asesino Neil Webb, verdadero lobo sanguinario de la novela, cuyo apetito voraz se colmará con las dos inocentes chicas Hannah y Yandirah, a las que irá a agredir y asaltar en la propia casa de Hannah. El policía Hernández hablando del criminal, confesará así: «Un asco, el tipo desde hace rato que le tenía hambre a la chiquilla » (p. 126). Para Amanda, la futura escritora, también es un recuerdo infantil el que introduce a su vez el tema del Mal:

Cuando era niña la casa de Mary Pat me daba miedo, tan grande, las ventanas detrás de las cuales no se veían señales de vida. En mis cuentos era la casa mala del barrio, una casa encallada en la arena que intimidaba a las otras a su lado y las de la hilera del frente, entre ellas la mía. Yo me contaba historias acerca de las casas antes de dormirme, relatos con moraleja incluida que hablaban de varias casas buenas enviadas al barrio de la casa de Mary Pat para aprender del Mal, para poder distinguirlo con claridad del Bien. A veces, después de unas semanas en su compañía, las casas descubrían cómo comportarse con corrección. Otras, la de Mary Pat se engullía a las demás. Era el horror. (p. 21) ${ }^{5}$

Así es como, en el imaginario infantil de la pequeña Amanda, « la casa de Mary Pat » llega a simbolizar el Mal, un mal que se expresa sintomáticamente al final bajo el signo de la voracidad ("se engullía a las demás »). La importancia fundamental de los cuentos en la estructuración de la personalidad de los niños y jóvenes adolescentes se observa también en el fluir de conciencia del niño Junior Webb, cuyo imaginario viene poblado por una inquietante figura de Abuelo criminal (que es en realidad la recreación infantil, narrativizada, del Abuelo real, padre de su propio padre Neil Webb):

Había una vez un Abuelo que vivía en una mansión abandonada y tenía una bola de cristal donde podía ver el mundo y sus alrededores. El Abuelo veía quién se portaba bien, por ejemplo Chris, que le había regalado su oso de peluche preferido a su hermano menor, y el Abuelo se acercaba a la casa de Chris, y tapiaba las puertas y cerraba las ventanas y ponía hollín en la chimenea y cerraba los desagües, en fi n, todo agujero que conectase a la casa con el mundo exterior. Y luego el Abuelo esperaba. Y llegaba la oscuridad. Y a la mañana siguiente, de Chris y su hermano y sus papás sólo quedaban huesos. (p. 80)

\section{El deporte y sus representaciones}

Otro elemento de la cultura popular estadounidense sutilmente subvertido en la novela es por supuesto el deporte, que se transforma poco a poco en signo inquietante. En efecto, el oso, en el primer capítulo es, ante todo, la inocente mascota de los Madison Bears, un famoso club de fútbol americano: "yo me secaba con una toalla roja con el logo de los Madison Bears " (p. 13), además de vincularse estrechamente, por cierto, con los cuentos y los chistes

\footnotetext{
${ }^{4}$ Las cursivas son nuestras.

${ }^{5}$ Las cursivas son nuestras.
} 
infantiles ${ }^{6}$ y los osos de peluche ${ }^{7}$. Después del doble asesinato de Hannah y Yandirah, Amanda analiza con cierto retraso el primer signo de mal agüero que constituía en realidad la mascota futbolística: «Al salir, ya cambiadas con nuestros buzos con el oso tonto de Madison High en el pecho -un oso grizzli amistoso, sé que esos osos son asesinos, he visto el documentalpapá nos pidió que nos tranquilizáramos » (p. 105). Y en la segunda parte de la novela, protagonizada por otro criminal (el Enterrador Colin), esta amenaza se concretará también justo antes del momento clave del asesinato de Christine:

Golpeé la puerta con insistencia hasta que, adolorido, logré abrirla. Me encontré, de pronto, en una habitación llena de luz, [...] una cama desatendida y Amanda, que se había quedado paralizada mirándome, terror en el rostro. Una polera roja con el oso de la escuela en el pecho le llegaba hasta los muslos. (p. 183) ${ }^{8}$

Ahora bien, en clave latinoamericana (y especialmente mexicana), lo mismo sucede con el símbolo de otro deporte y otra diversión -por cierto también altamente popular en los EE.UU.-, la máscara de lucha libre, que es primero un accesorio divertido para Junior (el hijo de Neil Webb), o sea un disfraz llamativo pero inocente: « [...] y sacamos las máscaras de un cajón. Tommy quería la azul, una de las que papá se trajo de México, de un tal Blue Demon, y yo me puse la negra [...] » (p. 31). Pero este doble signo de la cultura popular mexicana y estadounidense, muchas veces altamente paródico y burlón (para muchos la lucha libre es un deporte poco serio, aunque, eso sí, atlético), se revierte instantáneamente y se torna pronto signo de Maldad y accesorio potencial para las actividades criminales del padre violador, el inquietante señor Neil Webb: " [...] y yo me puse la negra, la que creo que se compró una vez que desapareció de Rota durante diez días y mamá estaba histérica » (p. 31).

Simbólicamente, la máscara del bien llamado Blue Demon llega pues a cristalizar, más que un simple juego de niños, la entrada al mundo de la violencia sexista y misógina y el descubrimiento de la auténtica Maldad: «[...] y encontré a papá enmascarado y gritando palabrotas, fuck you, fuck you, mamá tenía las manos atadas y lloraba, había marcas de golpe en la cara » (p. 31). En el sentido freudiano de la palabra, la escena primitiva estalla aquí en toda su violencia y abre paso al desencadenamiento de la violencia criminal del padre, que descubriremos a continuación. Pero hay más: la lucha libre, y la maniquea lucha que escenifica las más de las veces este deporte, también convoca una serie de referencias complementarias a la cultura popular cinematográfica, mediante el recuerdo de la mítica figura de (El) Santo (1917-1984), luchador profesional mexicano y protagonista de varias películas mexicanas clase Z durante más de veinte años, entre 1958 y 1982, desde Santo contra Cerebro del Mal (1958) hasta Santo vs el asesino de la televisión (1981), pasando precisamente por los famosos Santo contra Blue Demon en la Atlántida (1969), Santo y Blue Demon contra los monstruos (1969) o Santo y Blue Demon contra Drácula y el hombre lobo (1972).

\section{« And we'll have Halloween on Christmas »}

Si, para el niño y el lector occidental del hemisferio norte, la omnipresencia de la nieve en la novela (desde el epígrafe de Orhan Pamuk hasta las líneas finales atribuidas a Amanda) no deja de hacernos pensar a la vez en la muerte periódica de la naturaleza y en las fiestas navideñas, la fiesta que mejor caracteriza la novela es sin lugar a dudas Halloween, que los

\footnotetext{
${ }^{6}$ Véanse pp. 83-84.

${ }^{7}$ Sobre los osos de peluche en la novela, véanse primero, sobre el oso de Hannah, pp. 27, 87 (dos veces), 107-108 (dos veces) y 126; y también pp. 80, 130, 184 (donde aparecen otros osos de peluche). De hecho, en forma eminentemente sutil (y conformado por el imaginario infantil o adolescente), el eco entre la mascota futbolística y el retrato de una tal Lucy, primera novia compartida por los gemelos, unas líneas después, sugiere una visión del deseo sexual que ya contiene cierta dosis de peligrosidad (cazador/presa): « yo me secaba con una toalla roja con el logo de los Madison Bears. [...] Lucy era morena y tenía los ojos color miel » (p. 13).

${ }^{8}$ Las cursivas son nuestras.
} 
niños festejan durante la noche del 31 de octubre al $1^{\circ}$ de noviembre, o sea la noche previa a la fiesta cristiana de Todos los Santos (que precede a su vez al Día de Muertos). De hecho, el nombre Halloween es una alteración de All Hallows Eve(ning) (o sea la noche de todos los santos), y revela por lo tanto cómo esta fiesta pagana se articula secretamente con la fiesta cristiana de Todos los Santos. Aunque demasiado tarde, lo que reza -nunca mejor dicho- una de las canciones oídas en el entierro de Hannah y Yandirah, nos proporciona por supuesto una clave de lectura para la novela entera: And we'll have Halloween on Christmas (p. 133).

En efecto, ya en los primeros capítulos de la novela, dos narradores convocan esta fiesta folklórica y pagana, muy característica de la cultura anglosajona popular. El primero es Jem, profundamente traumatizado por la muerte accidental de su hermano gemelo:

El campo es extenso, la maleza está crecida, y hay calabazas por todas partes: grandes, redondas, ovaladas, pequeñas como una pelota de tenis, achatadas como si alguien las hubiera golpeado con un bate. [...] Luego vamos al laberinto con paredes de maíz y Tim se pierde, y nos preocupamos hasta que, de pronto, su voz chillona nos llama: está jugando con nosotros. Subimos a un camión con otros niños y sus padres, damos una vuelta por la granja, observamos los espantapájaros con cabeza de calabaza, algunos disfrazados de fantasmas y vampiros, otros de granjeros. [...] Entramos a una casa embrujada aferrados al brazo de papá; nos asustan una calavera de huesos crujientes, una bruja denariz verde. Tim, a punto de llorar, busca la salida. [...] La granja se llama The Singing Goat. Tenemos seis años. Ésos son mis primeros recuerdos de Tim. Han vuelto a mí una noche, en la oscuridad del gimnasio, sitio privilegiado de las apariciones de mi hermano. (p. 35)

Todo aquí apunta a la muerte, muerte ya efectiva o, por lo contrario, muerte por venir: muerte accidental de Tim, que convoca de hecho al recuerdo de Halloween (esta Noche de Muertos pagana y paródica); apariciones nocturnas y fantasmales del difunto en el gimnasio (que traen a la memoria los primeros recuerdos infantiles de Jem y las fiestas de Halloween); pero también visiones subliminales de un laberinto de maíz, en el que se esconde un posible monstruo depredador; una granja que anuncia la granja fatal del padre de Webb (donde éste se refugiará para violar y matar a las muchachas); un padre juguetón y protector (que se opone al padre violento, violador y asesino que es el señor Webb); y por fin los fantasmas y los vampiros, que recuerdan Vampirefreak (p. 28), el seudónimo del que se vale Webb para coquetear con Hannah en su página de MySpace, primera tentativa del cazador para acercarse a su presa. De forma que poco a poco, nos daremos cuenta de que Pensilvania (p. 53) puede rimar secretamente con Transilvania.

La segunda narradora que convoca la referencia a Halloween es Hannah (cuyo nombre comienza por cierto igual), es decir la que será pronto la víctima de las pulsiones criminales del señor Webb:

Una vez, en Halloween, mis papás me llevaron a ese barrio junto a mi amiga Rhonda; decían que allí se encontraban las mejores casas de la ciudad y los vecinos se tomaban muy en serio el trick o treat. Estacionamos cerca de una de las entradas principales y, bajo la luz anaranjada de unos faroles de sodio, caminamos por el medio de la calle, como hacían todos -calaveras festivas agarradas de la mano, alguien disfrazado del Joven Manos de Tijera y otro de Freddy Krueger, niños gritones con sobredosis de azúcar-. Mi bolsa estaba llena de dulces cuando la puerta de una casa con siluetas de brujas en las ventanas se abrió y apareció don Charlie; [...]. (pp. 41-42)

Después de la evocación de los recuerdos de Jem, esta segunda evocación de Halloween tiene varias funciones: amén de reintroducir esta fiesta folklórica que les encanta a los niños ${ }^{9}$, el episodio trae aquí otra figura paternal bonachona (don Charlie), que viene a completar el retrato inicial del entrenador Donald Walters, padre de la futura víctima del Enterrador, Christine (cap. 2). Sobre todo, el episodio viene a completar la serie de casas embrujadas (aunque sea paródicamente), como lo era en el relato de Amanda la casa de Mary Pat, sin

\footnotetext{
${ }^{9}$ Un chico en el caso de Jem, una chica en el caso de Hannah.
} 
revelar lo más importante: o sea que la casa del Mal puede ser a veces la casa de uno/una, en la que penetra inesperadamente un agresor (tan conocido como lo puede ser un simple vecino) y las auténticas fuerzas del Mal.

\section{De Halloween a Hollywood}

Esta nueva evocación de Halloween lleva consigo dos referencias cinematográficas: el Joven Manos de Tijera, héroe de la película Edward Scissorhands de Tim Burton, protagonizada por Johnny Depp (1990) y Freddy Krueger, héroe de una serie de películas exitosas creadas por Wes Craven (siendo la primera A nightmare on Elm Street, de 1984). En esta doble referencia, se observa una oposición entre un joven neo-romántico protagonizado por Johnny Depp (el Joven Manos de Tijera, que no encarna el Mal sino una diferencia monstruosa que genera aislamiento y melancolía) y Freddy Krueger, héroe cruel y excesivo y verdadera encarnación del Mal. Pero, esta incipiente antítesis axiológica Bien/Mal revelará pronto que el Mal es, en realidad, omnipresente y Yandirah, la segunda víctima del señor Webb, acabará horriblemente despedazada y descuartizada, como si la despedazara el mismo Joven Manos de Tijera.

Por ello, estrechamente relacionados con el imperio del cine adolescente, la novela convoca otros ritos sociales, propios de los jóvenes de las dos últimas décadas, como los pijama partys. Dice (o piensa) Hannah:

[...] podrían venir todas a dormir ese jueves. Un pijama party como habíamos tenido un par de veces. Esas reuniones nos unían; [...]. Luego veíamos un par de películas de horror tipo Saw y nos dormíamos entre risas. (p. 57)

En este contexto, para los personajes de la novela, incluso los más educados o leídos, como el periodista Daniel, una de las maneras más fáciles de abordar el Mal, y de entenderlo quizás, es remitir a sus representaciones más baratas popularizadas por el cine estadounidense: «Pensé en las palabras de Hernández, me imaginé una película clase B, una mezcla de horror y ciencia ficción. Monstruos que perseguían a la gente y al tocarla la convertían en fantasmas » (p. 126). Mediante el mismo procedimiento, el simple hecho de tomarse pastillas contra la depresión y la melancolía, en palabras de Hannah, suena pronto a película clase Z:

Recordaba cómo había sufrido con la muerte de Tim, cómo me había tirado a la cama la depresión (los llamaba Días de Zoloft, en honor a las pastillas que me había recetado el médico), y no quería que me volviera a ocurrir. (p. 58)

Todo funciona, pues, como si la representación del Mal fuera ahora inseparable de las múltiples representaciones popularizadas por la subcultura estadounidense -y ahora ampliamente mundializada - sin poder remitir explícitamente a los grandes mitos, sólo presentes en forma muy escueta en la novela (como lo recuerda de hecho en algunas entrevistas el mismo Edmundo Paz Soldán a propósito del mito de los gemelos, sutilmente convocado por el subtexto de la novela). Afirma con toda razón Giovanna Rivero: «Edmundo Paz Soldán se atreve a develar la profunda vocación Kitsch-romántica de la sociedad norteamericana, su afán por plastificarlo todo, ese deseo de cubrir con superficies tras superficies, piel tras piel, virtualidad tras virtualidad, clima contra clima, artificio sobre artificio, el emollo del asunto, sea éste emocional, político o económico ${ }^{10}$.

Los vivos y los muertos es pues una novela en que Virginia Woof (y no Virginia Woolf) sólo puede ser " una tienda de juguetes y comida para perros » (p. 16), y L'ultima Cena de Leonardo da Vinci, no una obra maestra del Renacimiento italiano, sino el pretexto para un best seller de Dan Brown -que sustituye hegemónicamente a la verdadera literatura en las librerías y los supermercados del mundo mundializado-. Un Dan Brown que convierte sus elucubraciones en verdades absolutas para el gran público:

Estaba [...] la reproducción de la Última Cena, había leído El código Da Vinci y me fijé y descubrí a María Madgalena entre los apóstoles. (p. 141)

\footnotetext{
${ }^{10}$ Giovanna Rivero, art. cit., p. 171.
} 
Asimismo, la única referencia literaria que aparece al final, para tratar de aprehender mejor la realidad confusa de las muertes múltiples de Madison, no es Edgar Allan Poe, ni Norman Mailer, ni Truman Capote, ni Dostoievski, sino Stephen King: «A alguien se le ocurrió [...] concluir que una maldición había caído sobre Madison High y sobre el pueblo. "Estamos viviendo en una novela de Stephen King”. No, las muertes en las novelas de King, incluso las más macabras, siempre forman parte de una trama con sentido " (p. 132). Novelas de Stephen King que, más allá de su formidable éxito de librerías, fueron llevadas a la pantalla en muchas ocasiones, dando a luz a películas que conforman una importante vertiente del trasfondo cultural de la novela de Paz Soldán: Carrie (1976), The Shining (1980), Christine (1983), Children of the Corn (1984), etc. ${ }^{11}$. Pero, como apunta Giovanna Rivero: « [...] un aspecto diferencial importantísimo entre "Los vivos y los muertos" y otras historias con similar tematización [...], es que esas novelas [o -añadimos- películas] no enfatizan los estereotipos como una forma de denunciarlos, es decir, de denunciar la maquinaria que los sostiene, porque están demasiado cerca, ya no los ven, no pueden verlos ${ }^{12}$.

La cuestión sería entonces ¿por qué, a pesar de la falta de referentes culturales tradicionales (obras maestras de la literatura, pintura, música, gran cine), carencia característica, por cierto, de la cultura estadounidense contemporánea en la que se mueven los protagonistas de la novela, el relato construye sin embargo efectos de sentido, simetrías, variaciones, trasmutaciones, que logran una densidad y una complejidad? La respuesta nos la proporciona tal vez la novela, al evocar la permanencia de los mecanismos de la psique humana y, sobre todo, la permanencia de los esquemas estéticos que conforman y dan a luz al verdadero arte:

La única clase que me entretuvo esa mañana fue la de Inglés. Miss BedfordLópez [...] habló de las comedias de Shakespeare y nos convenció de que sus estructuras seguían vigentes en películas como Virgen a los cuarenta años. (pp. 6061)

En una novela anterior de Edmundo Paz Soldán, Río Fugitivo (1998), se leía ya el mismo argumento en boca de otro profesor, apodado mister Macbeth, a propósito de los guionistas de las telenovelas que plagian a los clásicos: "los laberínticos conflictos familiares de las [telenovelas] mexicanas -hijos que se enamoran de sus madres, hermanas que sin saberlo se acuestan con sus hermanos- " no eran, según él, sino " "pedestres versiones de 'Edipo Rey' $"{ }^{13}$. Reescrituras o libres adaptaciones de los clásicos que son propios tanto de los teenage movies o de las películas hollywoodenses, claro está, como de la literatura hispanoamericana más canónica. Es lo que ejemplifica la famosa novela Cien años de soledad de Gabriel García Márquez (autor citado explícitamente un par de veces en Río fugitivo), que es como sabemos a la vez una reescritura tropical de Edipo Rey de Sófocles ${ }^{14} \ldots$ y un prequel un poco gore de Virgen a los cuarenta años: "Ya existía un precedente tremendo. Una tía de Úrsula, casada con un tío de José Arcadio Buendía tuvo un hijo [...] que murió desangrado después de haber vivido cuarenta y dos años en el más puro estado de virginidad porque nació y creció con una cola cartilaginosa en forma de tirabuzón y con una escobilla de pelos en la punta. Una cola

\footnotetext{
${ }^{11}$ En realidad, también se menciona a Lovecraft en el primer fragmento dedicado a Daniel, el periodista: «Quise leer los cuentos de Lovecraft, pero no pasé de las primeras diez páginas de "El horror de Dunwich” " (p. 111).

${ }^{12}$ Giovanna Rivero, art. cit., pp. 171-172.

${ }^{13}$ Edmundo Paz Soldán, Rio Fugitivo (Prólogo de Juan Gabriel Vásquez), Barcelona, Libros del Asteroide, 2008 ( $2^{\mathrm{a}}$ ed.), p. 52. La cita se hace de esta segunda edición (revisada por el autor). Véase al respecto nuestro artículo: « Del plagio y otro crímenes: Río fugitivo de Edmundo Paz Soldán », en Érich Fisbach (coord.), op. cit., pp. 81-94.

${ }^{14}$ Sobre este tema fundamental de Cien años de soledad, véase por ejemplo nuestro artículo "Insecto et incesto dans Cien años de soledad de Gabriel García Márquez: métamorphoses d’une anagramme », en Françoise Aubès (coord.), « Cien años de soledad » : fondations, héritages, crises, Paris, Ellipses, 2009, pp. 3147.
} 
de cerdo que no se dejó ver nunca de ninguna mujer [...] $»^{15}$. En todo caso, si las estructuras de las comedias de Shakespeare siguen vigentes en películas como Virgen a los cuarenta años, en Los vivos y los muertos, el subtexto sería más bien nada menos que Hamlet y su melancólico Príncipe de Elseneur, aunque el personaje de Colin el Enterrador también pueda remitir directamente a los dos sepultureros del acto V de la obra maestra de Shakespeare.

$\mathrm{Al}$ respecto, y llegado a este punto del análisis, creemos que una de las principales dificultades de la novela es que el lector llegue a tomar en serio todos los signos que proporciona la narración, por excesivos y evidentes que parezcan a primera vista. Tal es el caso, obviamente, del personaje de Colin, el Enterrador, que puede parecer demasiado estereotipado para que creamos, al principio, que puede llegar a cometer crímenes. Y sin embargo, lo hace, tal vez porque lo más probable y lo más evidente se confunde a veces con la realidad, y los criminales no tienen por qué ser psicópatas maquiavélicos que viven una doble vida y burlan con facilidad las investigaciones de la policía:

Siempre solitario y melancólico por los pasillos de la escuela, con su sobretodo negro tan sucio y raído de tanto que lo usaba, una vez alguien le había dicho que parecía el Enterrador de Madison. Él se rió y dijo me gusta el apodo, de ahora en adelante seré el Enterrador. Le gustaba la poesía de Alice Dickinson, series como $A$ dos metros bajo tierra, y sobre todo emo, esa música pop torturada, Dashboard Confessionnal, Jimmy Eat World, Thursday, Sunny Day Real State, Lots of Caulfield. (p. 72)

De forma que es cuando (y donde) menos se espera, cuando (y donde) la novela adquiere profundidad y complejidad, en los sutiles juegos de desdoblamientos y juegos de espejos con los estereotipos (el deportista seductor, la cheerleader sexy, el chico gótico, el periodista deprimido, etc.), como lo sugiere, de hecho, la misma novela: "A las chicas les gusta esa onda postgótica, de gótico que sabe que lo es y juega con él »(p. 72). Lo mismo podría decirse, por supuesto, de la novela, que juega sutilmente con una multiplicidad de códigos: sean literarios, narrativos, genéricos, cinematográficos, pictóricos u obviamente musicales.

Allí es donde las alusiones mainstream, los guiños de ojo, el plagio, las citas, y las citas de citas, llegan a conformar un complicadísimo entramado narrativo propio de la novelística postmoderna en la que se inserta, con gran eficacia, la obra de Edmundo Paz Soldán. Pero, una y otra vez, el medio y el fin siguen siendo la escritura, e inigualable es la capacidad de figuración y expresión del lenguaje literario. Es más: la complejidad del dispositivo narrativo elegido por el autor nos muestra que, bajo la superficie de la cultura mainstream, la técnica narrativa adoptada es de las más exigentes.

Por lo tanto, tendemos a discrepar de lo que pretende el Enterrador en la novela, que tiene con la profesora de inglés un apasionado debate sobre el alcance y la proyección de la cultura popular: "La profe de inglés dice que una cosa es poesía, otra las letras de una canción. El Enterrador le discutió: Bob Dylan ha sido postulado al Premio Nobel, no hay poeta más grande que él, todas las letras de sus canciones son poemas » (pp. 52-53). Debemos confesar que, como la profesora de inglés, y por conservadora que parezca la propuesta, abogamos modestamente por que les sigan otorgando el Premio Nobel de Literatura a escritores; como a Gabriel García Márquez en 1982, o más recientemente a Mario Vargas Llosa en 2010 o, dentro de una o dos décadas, por supuesto, a un escritor boliviano.

Benmiloud, Karim. "Insecto et incesto dans Cien años de soledad de Gabriel García Márquez : métamorphoses d'une anagramme », en Françoise Aubès (coord.), "Cien años de soledad » : fondations, héritages, crises, Paris, Ellipses, 2009, pp. 31-47.

Benmiloud, Karim. " Del plagio y otro crímenes: Río fugitivo de Edmundo Paz Soldán », en Érich Fisbach (coord.), Tradition et modernité dans l'œuvre d'Edmundo Paz Soldán, Angers, Presses de l’Université d’Angers, 2010, pp. 81-94.

${ }^{15}$ Gabriel García Márquez, Cien años de soledad (1967), Madrid, Cátedra (Letras Hispánicas), 2007, pp. 107-108. 
Fisbach, Érich (coord.). Tradition et modernité dans l'œuvre d'Edmundo Paz Soldán, Angers, Presses de l’Université d'Angers, 2010.

García Márquez, Gabriel. Cien años de soledad. Madrid, Cátedra (Letras Hispánicas), 2007 [ $1^{\mathrm{a}}$ ed. 1967]

Montoya Juárez, Jesús. « Ni apocalípticos ni integrados: medios audiovisuales en tres narradores del sur de América », Revista Iberoamericana, vol. LXXIII, núm. 221, oct.-dic., 2007, pp. 887-902.

Paz Soldán, Edmundo. Río Fugitivo (Prólogo de Juan Gabriel Vásquez), Barcelona, Libros del Asteroide ( $2^{\mathrm{a}}$ ed., revisada), 2008. [1 ${ }^{\mathrm{a}}$ ed. 1998].

Paz Soldán, Edmundo. Los vivos y los muertos, Madrid, Alfaguara, 2009.

Rivero, Giovanna. «El crimen Kitsch », en Érich Fisbach (coord.), Tradition et modernité dans l'œuvre d'Edmundo Paz Soldán, Angers, Presses de l’Université d’Angers, 2010, pp. 169-174. 